\title{
A Framework to Evaluate Blockchain Interoperability Solutions
}

\author{
Iulia Mihaiua $^{\mathrm{a}}$, Rafael Belchior ${ }^{\mathrm{b}, \mathrm{c}, *}$, Sabrina Scuri $^{\mathrm{d}}$, Nuno J. Nunes ${ }^{\mathrm{d}, \mathrm{b}}$ \\ ${ }^{a}$ Transilvania University of Brasov, Brasov, Romania \\ ${ }^{b}$ Department of Computer Science and Engineering, Instituto Superior Técnico, Universidade de Lisboa, Lisbon, Portugal \\ ${ }^{c}$ INESC-ID, Lisbon, Portugal \\ ${ }^{d}$ ITI / LARSyS, Funchal, Madeira, Portugal
}

\begin{abstract}
Decentralized ledger technology (DLT), in particular blockchain, is becoming ubiquitous in today's society. Just in the second quarter of 2021, centralized and decentralized exchanges had a volume of around $\$ 600$ billion. Enterprises are adopting this technology, following the opportunity to expand to new businesses. However, they need to connect their existing systems and processes to blockchains securely and reliably. Blockchain interoperability (BI) is emerging as one of the crucial features of blockchain technology. Fueled by the need to eliminate data and value silos, they realize the necessary bridge between centralized and decentralized systems.

As BI is still maturing, there are many unsolved challenges. In particular, it is still difficult for developers and practitioners to have control over processes spawning across several DLTs. In this report, we focus on the problem of managing cross-chain state in an integrated manner. First, we introduce the concept of cross-chain logic/cross-chain rules. After that, we present and discuss the results of our BI survey. Finally, we propose the BI evaluation framework, the first step to systematically evaluate BI solutions..
\end{abstract}

Keywords: Distributed Ledger Technology (DLT), Blockchain, Interoperability, Cross-chain transactions, Information visualization

\section{Introduction}

Enterprise systems that utilize blockchain-based applications and blockchain platforms might need a blockchain interoperability (BI) solution. If so, the solution needs several building blocks [1]. For example, identity portability is valuable across ledgers because it allows for managing identity across chains [2]. Likewise, data portability assumes a particular importance because it provides semantics to the data being transferred or migrated. Certain pieces of data in a blockchain can be interpreted as a blockchain view, carrying those semantics and proofs [?]. Finally, the systematic analysis of general-purpose cross-chain state is an unsolved problem. A systematic analysis of cross-chain state would allow to automatically derive crosschain logic rules among multiple interoperating blockchains and track several metrics (e.g., performance, end-to-to-end latency, energetic consumption). These measurements can provide the tools for developers to manage the life cycle of assets spawning across chains. Visualizing cross-chain transactions could also help to analyze and infer implicit business rules. One could inspect if cross-chain logic conforms to the defined business processes - and improve those rules, similarly to the area of process mining [3]. Analysis can identify bottlenecks, paving the way to improve performance and cutting costs.

\footnotetext{
${ }^{*}$ Corresponding Author:

Email address: rafael.belchior@tecnico.ulisboa.pt (Rafael Belchior )
}

Key Takeway 1. Benefits for practitioners and researchers

A cross-chain analysis is the application of data mining (in specific, pattern recognition) and process mining techniques to a heterogeneous, distributed, decentralized system, i.e., internet of blockchains [1]. Analyzing and visualizing crosschain transactions in such an environment can help stakeholders understand bottlenecks, identify processes, discover security issues, and provide more control over a BI solution.

The underlying research questions we aim to explore are i) What are cross-chain rules.1. and ii) What are the relevant metrics to assess cross-chain transactions and solutions? To better explore these research questions, we put forward preliminary research, where we surveyed experts in BI (people using and developing blockchain interoperability solutions) and nonexperts (people using and developing blockchain-based solutions). In summary, our contributions are:

- introduce the concept of cross-chain logic

- an analysis of the results on our survey regarding visualization and analysis of cross-chain transactions

- a framework to assess the performance of a BI solution

This report is organized as follows: Section 2 presents background on the cross-chain research area. Section 3 presents our

\footnotetext{
${ }^{1}$ another relevant research question is How to systematically analyse and visualize cross-chain rules? We defer this research question for future work.
} 
study. After that, we present a framework to evaluate the performance of a BI solution in Section 4. Finally, we present suggestions for future work and conclude the paper.

\section{Background}

From our previous work [1]: "A cross-chain transaction (CCTx), where "CC" stands for cross-chain, and "Tx" for transaction, is a transaction between different chains, which run the same runtime (homogeneous blockchains), for example, EVM-based blockchains. We use the CC-Tx, inter-chain transaction, and inter-blockchain transaction, and CB-Tx (crossblockchain transaction). A cross-chain decentralized application (CC-dApp) or a multiple blockchain decentralized application mDApp) is a distributed application that leverages crossblockchain transactions to implement its business logic. We consider a CC-Tx composed of atomic transactions on each ledger designed to accomplish a unit of work. The CC-Tx is an abstraction similar to distributed transactions in a two-phase commit system [4, 5]. For example, an asset transfer conducted via a cross-chain transaction is typically two transactions: one locking an asset on a source blockchain and another creating a representation of such assets in another blockchain.

Many solutions for BI already exist, including public connectors, hybrid connectors, and blockchain of blockchains, but there seems to be a lack of supporting tools to visualize, analyze, and evaluate BI solutions [1].

\section{Atomic Cross-Chain Transactions and Cross-Chain Rules}

Cross-chain transactions are distributed transactions occurring in an adversarial setting [6]. An atomic cross-chain transaction is a set of local blockchain transactions such that all are executed, or none is.

A cross-chain rule is a mapping taking a set of transactions from a source ledger to a set of transactions in a target ledger, i.e., an abstraction taking a set of triggers from a system that fires a set of actions on another. Cross-chain logic can trigger cross-chain transactions, which translate into local transactions on their respective blockchain. Multiple cross-chain rules running simultaneously are called cross-chain logic.

We exemplify this concept using the Carbon Emission Application from the Carbon Accounting and Certification WG, under Linux Foundation's Hyperledger Climate Action and Accounting SIG [7]. The Carbon Accounting and Certification WG aims to improve corporate carbon accounting by promoting transparency and accountability. To this end, a multiple blockchain approach is used ${ }^{2}$. This use case is implemented in Hyperledger Cactus [9], an open-source blockchain interoperability project (see Figure 1). This project utilizes a permissioned network, Hyperledger Fabric (Fabric), that gathers the energy used by corporations and converts it to emissions (Rule $\# 1)$. Then, these emissions are tokenized as emissions tokens

\footnotetext{
${ }^{2}$ the interested reader can follow up on 8
}

on the public Ethereum network (Rule \#2) so that one could trade emissions against allowances. When Rule \#1 is triggered, Cactus creates Tx1 (converting the energy into emissions) on Fabric. When Rule \#2 is triggered, Cactus creates Tx2 (tokenizing the emissions) on Ethereum. This combination of technologies allows maintaining some sensitive data private while publicly rewarding the participants.

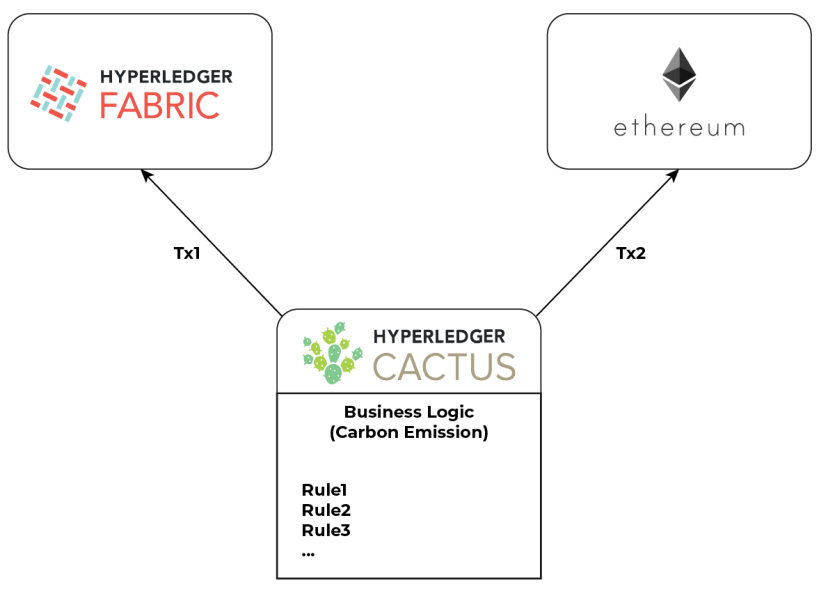

Figure 1: System supporting cross-chain logic between two blockchains

A formalization of cross-chain logic is left for future work.

\subsection{Methodology}

Analysis and visualization of cross-chain rules seem to be a key factor for enterprise adoption of multiple DLT approaches [2]. In order to develop a better understanding of what metrics and pieces of information are the most relevant for end-users when performing CC-Txs and gather insight on how to visualize these transactions effectively, we conducted a survey. Our survey targets both experts (i.e., individuals who have developed and/or managed - running and/or maintaining - a BI solution) and non-expert users. Specifically, the survey was pilottested with a group of 26 people. It comprised 17 items (openand closed-ended questions) and was structured into the following three main parts:

1. collection of demographic information, e.g., experience and knowledge of blockchain technology.

2. cross-chain transactions, comprising nine questions (five open-ended and four closed-ended). Those include experience with multiple blockchain DApps/Apps, understanding of cross-chain logics, relevant metrics, and information needs.

The survey was administered online for 24 days. A mixed approach (quantitative and qualitative) was used to analyze the data.

\subsection{Results}

A total of 26 individuals participated in the survey. Most of them were software developers $(n=9)$ and blockchain architects $(n=9)$. The professions of the remaining respondents included academic $(n=4)$, CEO/CTO $(n=3)$, investor $(n=3)$, and others 
$(n=4)$. Among them, 6 out of 26 respondents $(24 \%)$ are 'experts' - i.e., individuals who have developed and/or managed a BI solution. Most of the respondents in our sample are very experienced, with only five people having less than one year of blockchain experience. The experience of the remaining 21 individuals ranged from 2-3years $(n=6), 3$-5years $(n=10)$, and more than five years $(n=5)$. Projects developed by the expert group include protocols for verifiable data transfer between permissioned and permissionless blockchains and multi-chain payment channels. The non-experts group accounted for $76 \%$ of the population. However, the non-expert group at BI can be considered an expert group in the area of blockchain. Since the BI field is on its inception, we consider that the number of responses to our survey is significant.

The survey results indicate that there is no system in production to help users track cross-chain logic or gather and view cross-chain transaction metrics, a conclusion backed up by recent research [1]. Only 5 out of 26 people reported using general-purpose data traffic analysis systems (e.g., Grafana, Prometheus) to partially answer this need. Furthermore, eight respondents reported gathering metrics over the cross-chain logic. Five of them further detailed their answer by reporting which metrics they gathered. These are: total transactions (2 respondents), throughput (1 respondent), transaction status (1 respondent), transaction propagation time (1 respondent), latency ( 1 respondent), and time consumption - execution and communication - for each step (1 respondent).

Besides exploring the current practices - which are ultimately influenced by the existing software solutions- we wanted to dive deep into the information needs of our target users. For this reason, we have asked them to:

1. rank a set of seven metrics on a scale ranging from 1 (least important) to 5 (most important);

2. for those ranked as 'most important, provide a brief explanation of why such a metric is considered particularly relevant (optional); and

3. list additional metrics - i.e., not included in the previous question - that they would like to access and why.

\subsection{Discussion}

Contrary to expectations, $20 \%$ of the respondents indicated utilizing an interface to visualize cross-chain logic. Some include the usage of Prometheus to cross-analyze requests between servers. Although an excellent initial approach, the visualization could be limited to the base features of Prometheus. Supporting this data, $33 \%$ of the respondents actively track metrics of their cross-chain solutions, including the total transaction count, transaction status, transaction propagation time, latency (total and by step), and communication between components.

\section{Key Takeway 2. Trends on cross-chain}

A significant number of the respondents operate cross-chain solutions, tracking relevant metrics.
We asked the respondents to rank the importance of specific criteria (or metrics), using a Likert scale from 1 to 5 based on the relevance for their work on BI solutions(see Figure 3.2). All the seven metrics identified scored, on average, above 3 (on a 5-point scale). The average scores for end-to-end latency, end-to-end throughput, parties endorsing transactions, cross-chain logic, total transaction fees, carbon footprint, and energetic consumption were 3.692, 3.6, 3.5, 3.4, 3.3, 2.7, and 2.7. A $38 \%$ indicated that the most crucial metric is end-to-end latency of cross-chain transactions, while $31 \%$ indicated endto-end throughput as the most important feature. We hypothesize that the performance metrics (latency, throughput, fees) are more relevant to developers at this stage of the maturation of $\mathrm{BI}$, as opposed to qualitative metrics (energetic consumption, visualization of cross-chain logic, carbon footprint, endorsing parties).

On the second most important metric, 31\%, 35\%, and 38\% voted on parties endorsing the transaction, transaction fees, and the visualization of cross-chain rules. According to $27 \%$ of the respondents, the least important metric is the carbon footprint of cross-chain solutions.

\section{Key Takeway 3. Most important metrics}

Performance metrics, such as end-to-end latency, throughput, and cost (transaction fees), are currently the primary concerns for cross-chain analysis.

End-to-end latency and throughput are "what drives a better user experience which is the prerequisite of success in many cases". While qualitative metrics would be a prerequisite for a good experience for cross-blockchain middleware [5, 10, 11], performance metrics are essential since they are indicators of the security and the resilience to crashes [12] of the network. A trade-off between performance, cost, and a better user experience can and should be studied.

The visualization of cross-chain rules, namely the lifecycle of the cross-chain process, can provide insights into a solution's security and good operation. This would allow understanding, for instance, if finality has been achieved for a specific crosschain transaction (set of atomic transactions on their respective ledger).

\section{A BI Evaluation Framework}

In this section, we propose a simple framework to evaluate the performance of a BI solution based on the survey we conducted.

Since a CC-Tx is the sum of transactions issued to their respective ledgers, their performance metrics are related to each transaction.

The latency of a CC-Tx $(C \mathcal{T})$ composed by $n$ transactions is measured by the total latency of each transaction $\left(t_{1}, \ldots t_{n}\right)$, plus the coordination latency $c_{t}$ (employed by a BI solution that, for example, waits for finality, or rolls back transactions [5]). This is expressed in equation 1

$$
C \mathcal{T}_{t}=t_{1}+t_{2}+\ldots+t_{n}+c_{t}
$$




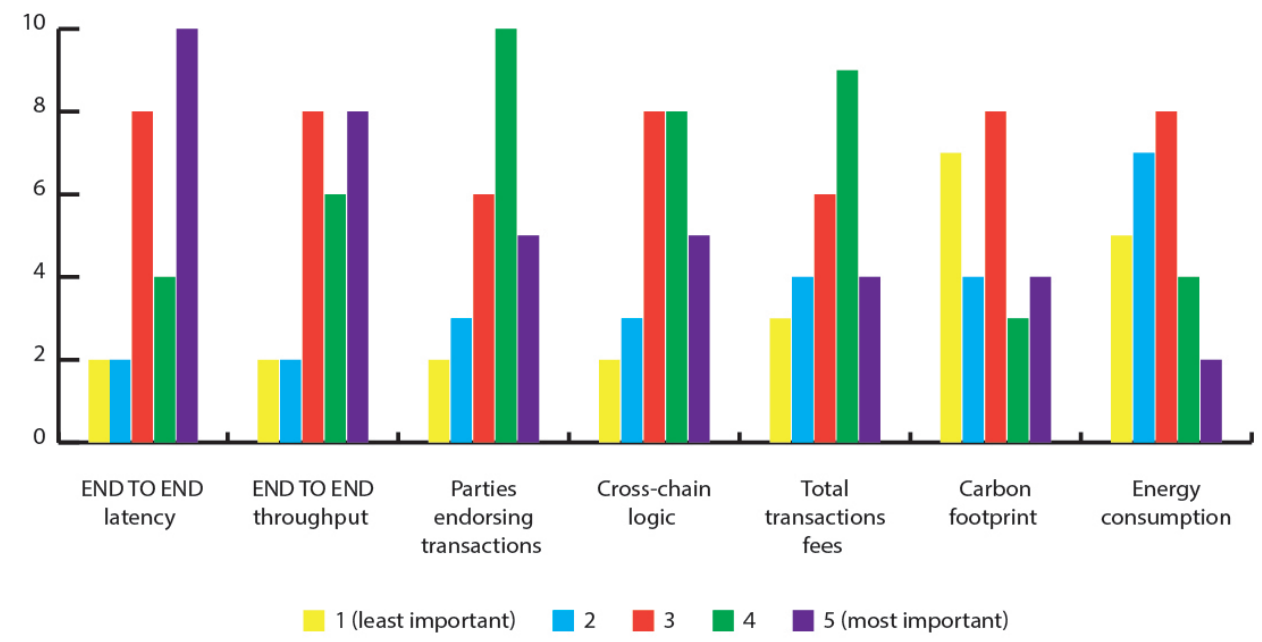

Figure 2: Results from the survey: weighted average ( 1 to 5 ) of each proposed cross-chain transaction metric

The throughput of $C \mathcal{T}$ composed by $n$ transactions is measured by the number of cross-chain transactions per time unit, as expressed by equation 2 .

$$
T=\frac{C \mathcal{T}}{t}
$$

Finally, the total cost (C) of $C \mathcal{T}$ is the sum of the cost of each sub-transaction $t_{i}$ :

$$
C=\sum_{i=1}^{n} \operatorname{cost}\left(t_{i}\right)
$$

\section{Key Takeway 4. Minimum Viable Evaluation Framework}

BI solutions can be evaluated using their end-to-end transaction latency, throughput, and cost.

\section{Future Work}

As future work, we will formalize the cross-chain logic and cross-chain state concepts. We will work towards creating an interface that allows us to visualize the most important metrics according to the respondents: end-to-end latency and throughput, total transaction fees, and visualization of crosschain rules. The code coming from this research will be publicly available at Hyperledger Cactus.

\section{Conclusion}

With BI solutions gaining popularity in academia and industry, there is a need to create supporting tools and evaluation frameworks. Supporting tools allow analysis and visualization of cross-chain transactions, providing end-users and developers tools to understand better their solutions in terms of throughput, cost, security, and asset life cycle.

In this paper, we started exploring the concept of cross-chain logic as the foundation for systematic cross-chain transaction analysis and visualization. Our preliminary survey showed potential to explore the end-to-end latency, end-to-end throughput, cross-chain logic metrics for academia and industry to explore this area. We propose a framework to evaluate blockchain interoperability solutions based on the collected criteria, providing the first step for systematically comparing BI solutions.

We pave the way for automatic visualization and analysis of cross-chain transactions. We hope that advances in this area remove barriers to the adoption of blockchain by enterprises.

\section{Acknowledgements}

This project has been supported by The Linux Foundation as part of the Hyperledger Summer Internships program under the Visualization and Analysis of Cross-chain Transactions project.

Rafael Belchior was supported by national funds through Fundação para a Ciência e a Tecnologia (FCT) with reference UIDB/50021/2020 (INESC-ID) and 2020.06837.BD. We thank all the respondents of our survey and the open-source community for supporting this work. We warmly thank Peter Somogyvari for valuable discussions on the topic and for providing support on Cactus. We thank Si Chen, Pritam Singh, Miguel Correia, André Vasconcelos, and Luke Riley for insightful discussions.

\section{References}

[1] R. Belchior, A. Vasconcelos, S. Guerreiro, M. Correia, A Survey on Blockchain Interoperability: Past, Present, and Future Trends ACM Computing Surveys 54 (8) (2021) 1-41. arXiv: 2005. 14282

URL http://arxiv.org/abs/2005.14282

[2] R. Belchior, PhD Thesis Proposal - Blockchain Interoperability, Tech. rep., Instituto Superior Técnico (sep 2021).

URL https://www.researchgate.net/publication/ 355370486_PhD_Thesis_Proposal

[3] W. M. Van Der Aalst, A. K. De Medeiros, Process Mining and Security: Detecting Anomalous Process Executions and Checking Process Conformance, Electronic Notes in Theoretical Computer Science 121 (SPEC. ISS.) (2005) 3-21. doi:10.1016/J.ENTCS.2004.10.013 
[4] R. Belchior, A. Vasconcelos, M. Correia, T. Hardjono, Enabling CrossJurisdiction Digital Asset Transfer, in: IEEE International Conference on Services Computing, IEEE, 2021.

[5] R. Belchior, A. Vasconcelos, M. Correia, T. Hardjono, HERMES: Fault-Tolerant Middleware for Blockchain Interoper\begin{tabular}{|l|lll|l|l}
\hline ability & TechrXiv 14120291/1 (mar 2021). & arXiv:1.
\end{tabular} 10.36227/TECHRXIV.14120291.V1

URL /articles/preprint/HERMES_Fault-Tolerant_ Middleware_for_Blockchain_Interoperability/14120291/1

[6] M. Herlihy, B. Liskov, L. Shrira, Cross-chain Deals and Adversarial Commerce Cross-chain Deals and Adversarial Commerce. PVLDB 13 (2) (2019) 100-113. doi:10.14778/3364324.3364326

URL https : //doi .org/10.14778/3364324.3364326

[7] Carbon Emission Working Group, hyperledger-labs/blockchain-carbonaccounting (2021).

URL https://github.com/hyperledger-labs/

blockchain-carbon-accounting

[8] Carbon Accounting and Certification WG, Carbon Accounting and Certification WG - Climate Action SIG - Hyperledger Foundation (2021).
URL https://wiki.hyperledger.org/display/CASIG/Carbon+ Accounting+and+Certification+WG

[9] H. Montgomery, H. Borne-Pons, J. Hamilton, M. Bowman, P. Somogyvari, S. Fujimoto, T. Takeuchi, T. Kuhrt, R. Belchior, Hyperledger Cactus Whitepaper (2020).

URL https://github.com/hyperledger/cactus/blob/master/ docs/whitepaper/whitepaper.md

[10] Quant Foundation, Overledger Network Whitepaper v0.3, Tech. rep., Quant (2019).

[11] M. Hargreaves, T. Hardjono, R. Belchior, Open Digital Asset Protocol draft 02 Internet-Draft draft-hargreaves-odap-02, Internet Engineering Task Force (2021).

URL

https ://datatracker.ietf .org/doc/html/ draft-hargreaves-odap-02

[12] R. Belchior, M. Correia, T. Hardjono, DLT Gateway Crash Recovery Mechanism draft 02 Internet-Draft draft-belchior-gateway-recovery-02, Internet Engineering Task Force (2021).

URL https://datatracker.ietf.org/doc/html/ draft-belchior-gateway-recovery-02 